\title{
Motivation zum Deutschlernen im tertiären Ausbildungsbereich. Ausgewählte Ergebnisse einer quantitativen Studie.
}

\section{Eliška Dunowski}

The paper is focused on L2-Motivation using as a specific example the motivation of students in the tertiary educational sphere to learn German as a foreign language. After a brief theoretical introduction, the results of a complex study are presented. The data was collected via an electronic questionnaire and statistically processed using SPSS and Statistica CZ to conduct a descriptive and factor analysis. The sample included 122 completed questionnaires, which corresponds to an approximate response rate of $35 \%$. The motivation to learn German, especially German for Academic or Specific Purposes, highly correlates to the relevance of the language as seen by the students.

extrinsic motivation - intrinsic motivation - integrative motivation - need for achievement relevance - German language for specific and academic purposes - questionnaire - factor analysis

Der Text setzt sich mit dem Thema der L2-Motivation auseinander, und zwar anhand des konkreten Beispiels der Motivation zum Deutschlernen im tertiären Bildungsbereich. Nach einer kurzen theoretischen Einleitung werden Ergebnisse einer quantitativen Studie präsentiert. Die Daten wurden mithilfe eines elektronischen Fragebogens erhoben und weiter mit SPSS und Statistica CZ im Rahmen einer deskriptiven Faktoren-Analyse statistisch ausgewertet. Die Stichprobe umfasste 122 komplett beantwortete Fragebögen, was einer Rücklaufquote von ca. 35 \% entspricht. Für die Motivation zum Deutschlernen, insbesondere auf der Ebene des akademischen und fachbezogenen Deutschlernens, ist das Motiv der Relevanz von zentraler Bedeutung.

extrinsische Motivation - intrinsische Motivation - integrative Motivation - Leistungsmotiv Motiv der Relevanz - fachbezogener und akademischer DaF-Unterricht - Fragebogen Faktorenanalyse

\section{Einleitung}

Der vorliegende Beitrag präsentiert Teilergebnisse einer Studie, die sich mit dem Thema der Motivation zum Fremdsprachenlernen beschäftigt und im Rahmen eines Dissertationsprojekts entstanden ist. Die Studie untersucht das multidimensionale Thema der Motivation unter dem Aspekt des fachbezogenen und akademischen Fremdsprachenunterrichts im tertiären Bildungsbereich. Als konkreter Ausgangspunkt diente die Konzeption des fachbezogenen und akademischen Fremdsprachenunterrichts an der Masaryk-Universität in Brünn, an der seit März 2006 jeder Absolvent eine Prüfung in einer Fremdsprache bestehen muss, wobei er/sie sich die konkrete Fremdsprache relativ frei aussuchen kann. An den meisten Fakultäten wird neben Englisch auch Deutsch angeboten, an 
manchen nur Englisch, an anderen wird außerdem noch Französisch, Spanisch oder Russisch als fachbezogene und akademische Fremdsprache unterrichtet. Die Tatsache, dass jede(r) Studierende der Masaryk-Universität ganz unabhängig von ihrem/seinem Studienfach eine Fremdsprachenprüfung bestehen muss, um sein/ihr Studium überhaupt erfolgreich abschließen zu können, ist unserer Ansicht nach ein sehr wichtiger Umstand, der sich in die Motivation zum Fremdsprachenlernen der Studierenden projiziert. Die Motivation zum Deutschlernen der Studierenden wird hier mithilfe eines gemischten Forschungsdesigns untersucht, wobei sowohl ein qualitatives als auch ein quantitatives Forschungsverfahren zum Einsatz kommt. Im Rahmen dieses Beitrags möchten wir die Ergebnisse des eher traditionellen quantitativen Forschungsverfahrens präsentieren, das mithilfe eines Fragebogens und statistischer Datenverarbeitung durchgeführt wurde. ${ }^{1}$

\section{Theoretische Grundlangen der L2-Motivationsforschung}

Motivation steht ungefähr seit den 50er Jahren des letzten Jahrhunderts im wissenschaftlichen Interesse der Fremdsprachendidaktik (Gardner und Lambert 1959). In den meisten L2-Motivationsstudien wird in der Regel mit folgenden drei Theorien gearbeitet: 1 . Die der integrativen und instrumentellen Motivation und der Rolle der Einstellung und Orientierung von Gardner (2005), 2. die der Rolle der Selbstbestimmung (self-determination) aus der Selbstbestimmungstheorie (Deci und Ryan 1993) und 3. die der Rolle des Erfolgs (oder Misserfolgs) aus der Attributionstheorie (z. B. Williams, Burden und Al-Baharna 2001). Auch unsere Studie basiert hauptsächlich auf diesen Theorien, die nun kurz vorgestellt werden (vgl. Dunowski 2015).

\subsection{Gardners Beitrag zur Motivationsforschung beim Lernen einer L2}

Die ersten empirischen Studien, die sich mit der L2-Motivation intensiv auseinandergesetzt haben, wurden im bikulturellen Raum der kanadischen Metropole Montreal von R. C. Gardner und seinen Kollegen durchgeführt (1959). Die grundlegende und bis heute ausgiebig diskutierte Aussage ist vor allem seine Unterscheidung von integrativer und instrumenteller Motivation: Integrativ motiviert ist der Lerner dann, wenn es sein Ziel ist, sich der Gesellschaft zu nähern, deren Sprache er lernt (Gardner 2005: 7). Instrumentell motiviert ist dagegen ein Lerner, der eine Fremdsprache aus einem pragmatischen Grund lernt, z. B. um eine Prüfung

1 Der Beitrag präsentiert nur einen Teil einer komplexen Studie, die aus zwei unabhängigen Forschungsphasen besteht: einer qualitativen Forschungsphase, in der aufgrund eines semi-offenen Interviews Daten erhoben wurden und individuelle Motivationsprofile erstellt wurden, und einer quantitativen Forschungsphase, in der Daten mithilfe eines elektronischen Fragebogens erhoben wurden, deren ausgewählte Ergebnisse im Rahmen dieses Beitrags präsentiert werden. 
abzulegen oder eine höhere berufliche Qualifikation zu erwerben (vgl. Rost-Roth 2010: 877). Der ursprüngliche Ausgangspunkt für diese Unterscheidung wurde von O. Mowrer (1942) übernommen. Dieser geht davon aus, dass eine Identifizierung mit der oder Annäherung an die Gesellschaft, in der die gelernte Sprache gesprochen wird, notwendig für den eigentlichen Spracherwerb ist.

\subsection{Die Selbstbestimmungstheorie (self-determination theory)}

Gardners Differenzierung von integrativer und instrumenteller Motivation ähnelt der Unterscheidung zwischen extrinsischer und intrinsischer Motivation, die im Rahmen der Selbstbestimmungstheorie getroffen wird. Die extrinsische Motivation richtet sich auf diverse äußere Bedingungen, z. B. verschiedene Formen von Sanktion und Belohnung. Die intrinsische Motivation entspringt dagegen dem rein subjektiven Interesse am Lernen oder an der Lösung der selbst gestellten Aufgabe.

Die Selbstbestimmungstheorie entwickelte sich im Rahmen der kognitiven Psychologie in den 1980er Jahren und ist hauptsächlich mit den Namen von E. L. Deci und R. M. Ryan (vgl. Deci und Ryan 1993: 223) verbunden. Die Selbstbestimmungstheorie unterscheidet zwischen autonomer und kontrollierter Motivation, wobei die autonome Motivation sowohl extrinsische als auch intrinsische Motivation umfasst, wenn diese mit den inneren Werten des Lerners übereinstimmt. Kontrollierte Motivation entspricht dann der extrinsischen Motivation. Allgemein gilt die intrinsische Motivation als tiefer und dadurch auch wertvoller. Eine Reihe von kleineren Studien zeigt jedoch, dass starke extrinsische Motivation die intrinsische Motivation sowohl schwächen als auch stärken kann. Geschwächt werden kann die intrinsische Motivation vor allem dann, wenn der Lerner über ungenügend Selbstbestimmung (also Autonomie und Kontrolle) in Bezug auf die zu lösende Aufgabe verfügt. Sollte der Lerner jedoch über genug Selbstbestimmung verfügen, kann dauerhafte starke extrinsische Motivation auch zur Stärkung der intrinsischen Motivation führen (vgl. Kirchner 2004).

\subsection{Attributionstheorie}

Die zweibahnige Wirkung von Motivation und Erfolg (bzw. des Lernfortschritts) ist schon seit den 1980er Jahren wissenschaftlich belegt. Gerade die subjektive Wahrnehmung des eigenen Erfolgs oder Misserfolgs und ihr Zusammenhang mit Motivation liegt im Forschungsinteresse der Attributionstheorie (vgl. Riemer 2010: 1152). Ob jedoch Motivation für Erfolg verantwortlich ist oder Erfolg für Motivation, ist bisher umstritten. Hermann (1978) zeigt in ihrer Studie, dass Erfolg die Motivation (bzw. Orientierung und Einstellungen) des Lerners positiv beeinflusst. Im Sinne „dieser Wirkungsrichtung“ formuliert Hermann die Resultativhypothese, die das Gegenteil der sogenannten Kausalhypothese ist. Laut dieser führt ein höheres 
Maß an Motivation auch zu größerem Erfolg. Hermanns Ergebnisse stehen allerdings nicht im Widerspruch zu der Kausalhypothese und wurden auch durch andere Studien unterstützt, z. B. Burstall 1975 (vgl. Riemer 1997: 20-24).

Neben den L2-Motivationstheorien geht unsere Studie von der theoretischen Grundlage des fachbezogenen und akademischen Fremdsprachenunterrichts aus. In diesem Fall beziehen wir diesen eigenen Terminus auf das Englische EAP und ESP bzw. English for academic purposes und English for specific purposes (Hutchinson und Waters 1987). In der deutschen Fachliteratur wird dieses Thema von H. Funk (2009) behandelt, der sich hauptsächlich zum berufsbezogenen Fremdsprachenunterricht äußert. Dieser Begriff entspricht jedoch unserem Verständnis der Problematik nicht ganz, da wir uns im Rahmen unserer Studie ausschließlich dem tertiären und dadurch auch akademischen Fremdsprachenunterricht widmen. Aus diesem Grund benutzen wir hier den Begriff fachbezogener und akademischer Fremdsprachenunterricht.

\section{Zur Durchführung der Untersuchung}

\subsection{Die Datenerhebung}

Unser Forschungsinteresse ließe sich in folgender Forschungsfrage formulieren: Wie ist die Motivation der Studierenden der Masaryk-Universität zum allgemeinen und zum fachbezogenen und akademischen Deutschlernen? Das quantitative Forschungsverfahren basierte auf einer Datenerhebung mit Hilfe eines elektronischen Fragebogens, der hauptsächlich durch zwei andere Fragebögen zum Thema der Motivation zum Deutschlernen inspiriert wurde (vgl. Schlack 2003 und Rahamaliarison 2009). Zur Datenerhebung nutzten wir die Software Quest Back, die wir unter www.unipark.com erworben haben. Zur statistischen Bearbeitung der Daten diente uns die Analyse-Software SPSS 22 sowie Statistica CZ.

Der Fragebogen bestand aus insgesamt 41 Items, die auf 3 Fragebatterien verteilt worden waren. ${ }^{1}$ Die geschlossenen Fragen konnten die Probanden mithilfe einer fünfstufigen Likert-Skala beantworten $(1=$ ich stimme voll $\mathrm{zu}, 5=$ ich stimme gar nicht zu). Die durchschnittliche Bearbeitungszeit betrug etwas mehr als 10 Minuten und am Ende der Datenerhebung, die im Frühjahr 2015 stattfand, hatten wir 122 komplett beantwortete Fragebögen zur Verfügung, was einer Rücklaufquote von fast $35 \%$ der kompletten Stichprobe entspricht. Der Fragebogen arbeitete ohne vorher formulierte Hypothesen, um eventuell vorhandene weitere Motivationsfaktoren nicht von vorne herein auszuschließen.

1 Die Finalversion des Fragebogens entsprach einem großen Zeit- und Arbeitsaufwand, der selbstverständlich mit Pilotierung und diversen Fachdiskussionen verbunden war sowie der fachlichen Opponentenkritik gestellt wurde. 


\subsection{Die Befragten}

Unsere Untersuchungsteilnehmer waren Studierende der Masaryk-Universität aus vier Fakultäten, ${ }^{1}$ in denen im Rahmen des fachbezogenen und akademischen Fremdsprachenunterrichts auch Deutsch angeboten wird. Die Probandenauswahl stützte sich auf ein Kriterium: Um Sprachniveau und Kursbedingungen konstant zu halten, mussten die Probanden zur Zeit der Datenerhebung in einem Master-Studiengang eingeschrieben sein. ${ }^{2}$ Wie bereits erwähnt haben insgesamt 122 Studierende den Fragebogen beantwortet, was ca. $35 \%$ der kompletten Stichprobe entspricht. Darunter waren 83 Frauen und 39 Männer im Alter von 22 bis 28 Jahren.

\section{Ausgewählte Ergebnisse der Umfrage}

Die Datenanalyse verlief auf zwei Ebenen: Zuerst wurde, wie üblich, eine deskriptive Analyse durchgeführt, die einen ersten Einblick in die Daten ermöglichte. Diese Analyse zeigte jedoch keine besonders interessanten oder im Hinblick auf unser Forschungsinteresse relevanten Ergebnisse. Auf der zweiten Ebene wurde eine Faktorenanalyse durchgeführt, die zeigte, dass wir statt mit 41 Items aus dem Fragebogen oder zehn Motivkategorien (die durch die Items untersucht wurden) nur mit vier latenten Variablen, den so genannten Faktoren, weiterarbeiten konnten.

Der erste Faktor erfasste die Motive im Bereich der knowledge-orientation (vgl. Clément und Kruidenier 1983) und des Ehrgeizes. Diese Motivgruppe beschreibt eine Situation, in der der Lerner zum Lernen der Fremdsprache motiviert ist, weil er seine bereits erworbenen Fremdsprachenkenntnisse erweitern und dadurch seinen Ehrgeiz befriedigen möchte. Wie auf der folgenden Abbildung (Abb. 1) zu sehen ist, bewegen sich die Werte für die Antworten der Probanden an allen vier Fakultäten zwischen 2,2 und 2,8, d. h. im Bereich der Zustimmung der vorgegebenen Likert-Skala. Das würde darauf hindeuten, dass diese Motivgruppe (Ehrgeiz und knowledge-orientation) für die Probanden aller Fakultäten wichtig ist, am meisten für die Probanden der Philosophischen und der Sozialwissenschaftlichen Fakultät (die gemeinsamen Fremdsprachenunterricht haben und daher im Rahmen der Studie als eine Fakultät betrachtet werden) und am wenigsten für die Probanden der Pädagogischen Fakultät, die eher zu dem Wert 3 neigen.

1 Die vier konkreten Fakultäten, an denen auch Deutsch im Rahmen des fachbezogenen und akademischen Unterrichts angeboten wird, sind: die Philosophische Fakultät und die Sozialwissenschaftliche Fakultät, die zur Zeit der Datenerhebung gemeinsamen Fremdsprachenunterricht hatten (weshalb wir beide Fakultäten bei der Auswertung als eine Fakultät betrachten), weiter die Pädagogische Fakultät, die Juristische Fakultät und die Wirtschaftswissenschaftliche Fakultät.

2 Studierende in einem Bachelor-Studiengang müssen an allen Fakultäten der Masaryk-Universität eine Prüfung in einer fachbezogenen und akademischen Fremdsprache auf dem Niveau B1 des Gemeinsamen Europäischen Referenzrahmens bestehen, Studierende in Master-Studiengängen auf dem Niveau B2. 


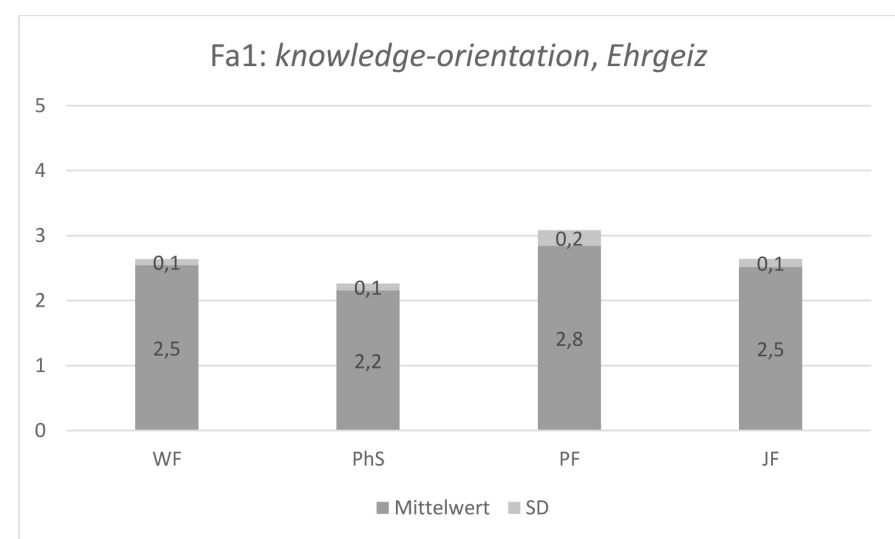

Abb. 1: Faktor 1: Motiv im Bereich Ehrgeiz und knowledge-orientation

Der zweite Faktor erfasst die Motive im Bereich travel-und friendship-orientation und Motive im Sinne der integrativen Motivation (Gardner 2005) und des Kontakts mit der Zielsprache (vgl. Riemer 1997). Wie aus Abb. 2 zu entnehmen ist, neigen alle vier Gruppen zu dem Wert 3, der nur bei Probanden der Wirtschaftswissenschaftlichen Fakultät überschritten wird $(3,1)$. Das bedeutet, dass diese Motivgruppe für die Probanden der Wirtschaftswissenschaftlichen Fakultät eher unbedeutend ist. Für die Probanden der Juristischen Fakultät sind die entsprechenden Motive im Vergleich zu den Probanden anderer Fakultäten von größerer Bedeutung $(2,7)$, wobei allerdings nur geringe Unterschiede zu den Probanden der Philosophischen und Sozialwissenschaftlichen Fakultät (2.8) sowie den Probanden der Pädagogischen Fakultät bestehen.

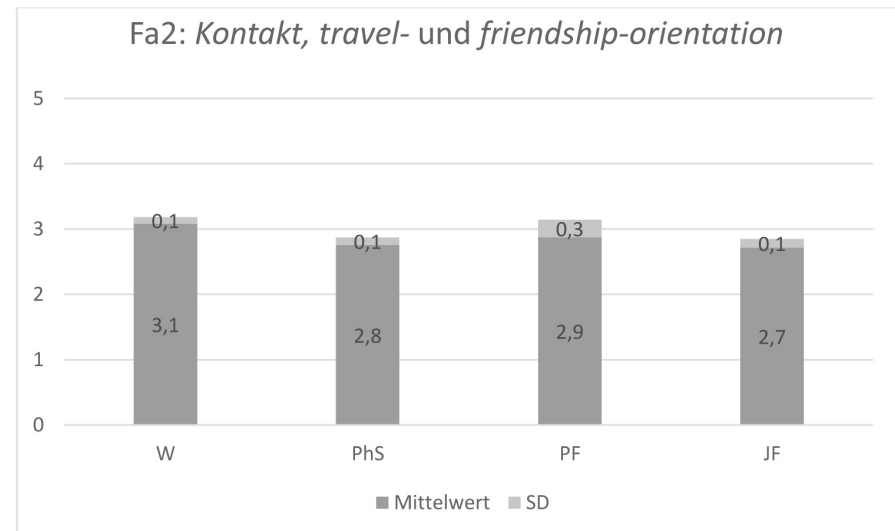

Abb. 2: Faktor 2: Integrative Motive und Kontakt 
Der dritte Faktor erfasst alle Motive, die die deutsch-tschechischen Beziehungen aus geographischer, historischer, kultureller, politischer und wirtschaftlicher Perspektive berühren. Auf Abb. 3 ist zu sehen, dass diese Motivgruppe für die Probanden aller Fakultäten von Bedeutung ist (Werte der Zustimmung zwischen 2,1 und 2,7). Am bedeutendsten sind sie für die Probanden der Juristischen Fakultät $(2,1)$, es folgen die Pädagogische Fakultät $(2,5)$, die Philosophischen und die Sozialwissenschaftliche Fakultät $(2,6)$ und schließlich die Wirtschaftswissenschaftliche Fakultät $(2,7)$.



Abb. 3: Faktor 3: Motive hinsichtlich der deutsch-tschechischen Beziehungen

Der letzte Faktor erfasst hauptsächlich das Leistungsmotiv (vgl. need for achievement, Dörnyei 1994), das mit dem Bedürfnis nach Erfolg zusammenhängt. Auf Abb. 4 ist zu erkennen, dass bei diesem Motiv alle Werte zwischen 2,5 und 3 liegen. Das würde eher auf eine geringere Bedeutung des Leistungsmotivs hindeuten. Die geringste Bedeutung messen diesem Motiv die Probanden der Wirtschaftswissenschaftlichen Fakultät bei $(3,0)$, gefolgt von den Probanden der Philosophischen und der Sozialwissenschaftlichen Fakultät sowie der Juristischen Fakultät (beide 2,8). An der Spitze stehen die Probanden der Pädagogischen Fakultät, die diesem Motiv die größte Relevanz beimessen. 


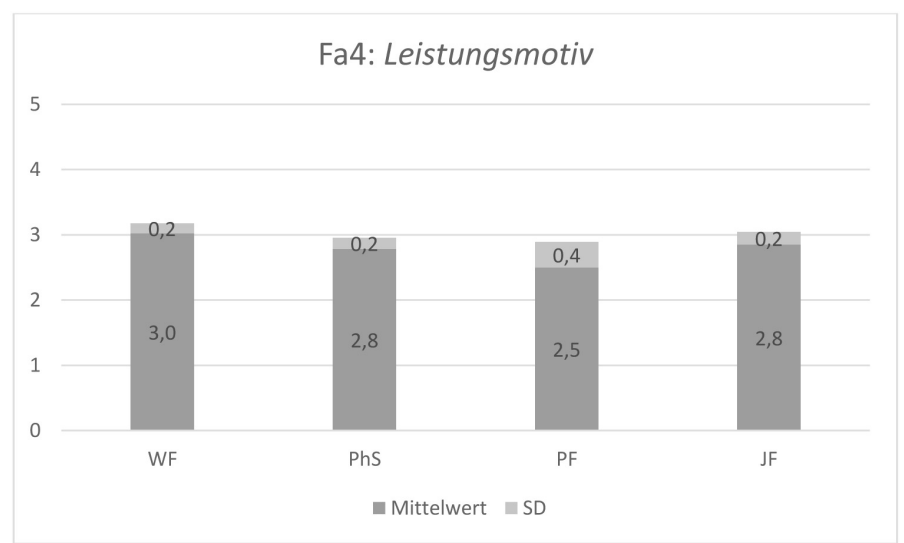

Abb. 4: Faktor 4: Leistungsmotiv

Die Ergebnisse haben wir im Rahmen diverser statistischer Tests weiter untersucht, besonders unter dem Aspekt der Unterschiede zwischen den Antworten der Probanden der einzelnen Fakultäten. Die Tests ANOVA, Bonferroni-Methode und Turkeys HSD-Test ${ }^{1}$ haben leider keine statistisch signifikanten Unterschiede gezeigt. Nur Fishers LSD-Test ${ }^{2}$ bestätigte statistisch signifikante Unterschiede zwischen den Probanden der einzelnen Fakultäten im Rahmen der ersten drei Faktoren. Im Rahmen des vierten Faktors, der das Leistungsmotiv erfasst, wurden zwischen den Probanden der einzelnen Fakultäten keine statistisch relevanten Unterschiede festgestellt.

\section{Fazit}

Die Ergebnisse der Faktorenanalyse und der weiter durchgeführten statistischen Tests zeigen, dass es statistisch signifikante Unterschiede in der Motivation der Probanden der einzelnen Fakultäten gibt. Hinsichtlich des Motivs der knowledgeorientation und des Ehrgeizes wurde ein Unterschied zwischen den Probanden der Philosophischen und der Sozialwissenschaftlichen Fakultät (die gemeinsamen Fremdsprachenunterricht haben und daher als eine Gruppe betrachtet werden) und den Probanden der anderen Fakultäten deutlich, was sich beinahe klischeehaft durch das allgemeine Interesse an Wissen und Fremdsprachenkenntnissen der Probanden der ersten Gruppe erklären ließe.

1 Es handelt sich um diverse datenanalytische und statistische Verfahren, die Varianzen und Prüfgrößen berechnen, um Aufschlüsse über die hinter den Daten steckenden Gesetzmäßigkeiten zu ermitteln.

2 Fishers LSD-Test (Least Significant Difference Test) beruht auf dem Zweistichprobent-Test, jedoch wird die Varianz mit Hilfe aller Gruppen berechnet. Dieser Test wird zum Durchschnittsvergleich zweier Datensätze benutzt. 
Die Ergebnisse zum zweiten Faktor (integrative Motivation) zeigen deutlich, dass es einen signifikanten Unterschied zwischen den Probanden der Wirtschaftswissenschaftlichen Fakultät und den Probanden der Philosophischen und der Sozialwissenschaftlichen Fakultät sowie den Probanden der Juristischen Fakultät gibt, wobei sich in diesem Fall gerade die integrative Motivation als eher unbedeutend für unsere Probanden erweist. Dieses Ergebnis führt uns nunmehr auf die Ebene des fachbezogenen und akademischen Deutschlernens, und zwar in dem Sinne, dass gerade Studierende der Wirtschaftswissenschaftlichen Fakultät fast ausschließlich zum Lernen von Fachdeutsch motiviert sind, sodass die integrative Motivation für sie von sehr geringer Bedeutung ist. Dies lässt sich weiter dadurch erklären, dass gerade Probanden der Wirtschaftswissenschaftlichen Fakultät, die über gute Deutschkenntnisse verfügen, einen großen Vorteil mit in ihren zukünftigen Beruf mitnehmen. Hiermit wird auch die Bedeutung des Motivs der Relevanz (relevance, Dörnyei 1994) deutlich, welches sich schon in diesem zweiten Faktor zeigt. Gemeint ist damit, dass die Studierenden zum Deutschlernen motiviert sind, weil der Unterrichtsinhalt für sie relevant ist. Gerade dieses Motiv zeigt sich auch im Rahmen des folgenden Faktors als entscheidend. Die Relevanz (im Sinne von Dörnyei 1994) einer Fremdsprache geht in diesem Fall also auf Kosten der integrativen Motivation.

Eine ähnliche Wechselwirkung zwischen verschiedenen Motivationsarten zeigt sich auch beim dritten Faktor, der das Interesse an den deutschtschechischen Beziehungen darstellt. Hier zeigen sich statistische Unterschiede zwischen den Probanden der Juristischen Fakultät auf der einen, und den Probanden der Philosophischen und der Sozialwissenschaftlichen sowie der Wirtschaftswissenschaftlichen Fakultät auf der anderen Seite. Dieses Ergebnis lässt sich hauptsächlich durch die gemeinsame deutsch-tschechische Geschichte erklären, die besonders im juristischen Bereich bis heute zu spüren ist, und weist hiermit wiederum auf das bereits erwähnte Motiv der Relevanz im Rahmen des fachbezogenen und akademischen Fremdsprachenunterrichts hin.

Der vierte Faktor, das Leistungsmotiv, erweist sich als eher unbedeutend und weist keine statistisch signifikanten Unterschiede zwischen den Probanden der verschiedenen Fakultäten auf, wodurch leider auch wenig Möglichkeit für Interpretationen bleibt. Eines wird jedoch deutlich: Gerade bei diesem Motiv erweist es sich als schwierig, ausschließlich mit quantitativ gewonnenen Daten und Ergebnissen zu arbeiten. Der hier beschriebenen quantitativen Studie folgte jedoch eine qualitative Studie, die Unterschiede zwischen den Studierenden einzelner Fakultäten im Rahmen dieses Motivs nachwies (vgl. z. B. Dunowski 2015). Der Vergleich dieser beiden Ergebnisse lässt sich dann durch zwei Tatsachen erklären. Einerseits ist dies der hohe Grad an Subjektivität, der beim Leistungsmotiv vorhanden ist und dadurch signifikante statistische Ergebnisse verhindert, andererseits ist dies der Charakter des Forschungsthemas, die L2-Motivation. Traditionell wird das 
Thema mit quantitativen Forschungsverfahren bearbeitet (vgl. z. B. Gardner 2005; Rahamaliarison 2006; Schlak 2003), es wird jedoch immer deutlicher, dargelegt vor allem in Riemer $(1996,2004)$, dass die L2-Motivationsforschung auch qualitative Methoden braucht.

Selbst wenn es scheinen mag, dass manche Ergebnisse nur allgemein bekannte und zu erwartende Annahmen bestätigen, muss betont werden, dass diese Annahmen nun durch eine Studie wissenschaftlich untermauert werden konnten. Für die Ebene des fachbezogenen und akademischen Deutschlernens zeigte sich als Schlüsselmotiv das Motiv der Relevanz, das auf den geographischen, wirtschaftlichen und historischen deutsch-tschechischen Beziehungen beruht. Darüber hinaus zeigen die Ergebnisse aber auch, wie subjektiv, dynamisch und kompliziert das Phänomen der L2-Motivation ist. Diese Attribute nehmen ihm jedoch nichts an seiner Attraktivität sowohl für die Forschung als auch für die Praxis.

\section{Literaturverzeichnis}

Burstall, Clare (1975): Factors affecting foreign language learning: A consideration of some recent research findings. In: Language Teaching \& Linguistic Abstracts 8/1975, S. 5-21.

Clément, Richard / Kruidenier, Bastian G. (1983): Orientations in second language acquisition: The effects of ethnicity, milieu, and target language on their emergence. In: Language Learning 33/3/1983, S. 273-291.

Deci, Edward L. / Ryan, Richard M. (1993): Die Selbstbestimmungstheorie der Motivation und ihre Bedeutung für die Pädagogik. In: Zeitschrift für Pädagogik, 39/2/1993, S. 223-238.

Dörnyei, Zoltán (1994): Motivation and motivating in the foreign language classroom. In: The Modern Language Journal 78/1994, S. 273-284.

Dunowski, Eliška (2015): Motivation im fachbezogenen DaF-Unterricht bei tschechischen Studierenden: erste Ergebnisse einer qualitativen Studie. Brno, Tribun. S. 108-123.

Funk, Hermann (2007): Berufsbezogener Fremdsprachenunterricht. In: Bausch, Karl-Richard / Christ, Herbert / Krumm, Hans-Jürgen (Hg.), Handbuch Fremdsprachenunterricht. Tübingen, A. Francke Verlag. S. 175-179.

Gardner, Robert C. / Lambert, Wallace E. (1972): Attitudes and motivation in second language learning. Rowley. Newbury House Publishers.

Gardner, Robert C. (2005): Integrative motivation and second language acquisition. Verfügbar unter: http://publish.uwo.ca/ - gardner/docs/caaltalk5final.pdf (16. 2. 2016).

Hutchinson, Tom, \& Walters, Alan (1987): English for Specific Purposes. Cambridge University Press.

Kirchner, Katharina (2004): Motivation beim Fremdsprachenerwerb: Eine qualitative Pilotstudie zur Motivation schwedischer Deutschlerner. In: Zeitschrift für Interkulturellen Fremdsprachenunterricht, 9/2/2004. Verfügbar unter: http://www.ualberta.ca/-german/ ejournal/Kirchner2.htm (16. 2. 2016).

Rahamaliarison, Hanitrarivelo Olivia (2009): Motivation im DaF-Unterricht in Madagaskar. In: Info DaF 36/4/2009. S. 340-355.

Riemer, Claudia (1997): Individuelle Unterschiede im Fremdsprachenerwerb. Die Wechselwirksamkeit ausgewählter Einflussfaktoren. Hohengehren. Schneider Verlag. 
Riemer, Claudia (2004): Zur Relevanz qualitativer Daten in der neueren L2Motivationsforschung. In: Börner, Wolfgang / Vogel, Klaus (Hg.) Emotion und Kognition im Fremdsprachenunterricht. Tübingen. De Gruyter. S. 35-65.

Riemer, Claudia (2010): Motivierung. In: Krumm, Hans-Jürgen / Fandrych, Christian / Hufeisen, Britta / Riemer, Claudia (Hg.) Deutsch als Fremd- und Zweitsprache. Ein internationales Handbuch. Band 1. Berlin. De Gruyter. S. 1151-1156.

Rost-Roth, Maria (2010): Affektive Variablen/Motivation. In: Krumm, Hans-Jürgen / Fandrych, Christian / Hufeisen, Britta / Riemer, Claudia (Hg.) Deutsch als Fremd-und Zweitsprache. Ein internationales Handbuch. Band 1. Berlin. De Gruyter. S. 875-884

Schlag, Bernhard (2009): Lern- und Leistungsmotivation. Wiesbaden. Springer.

Schlak, Thorsten / Banze, Kathrin / Haida, Justine / Kilinc, Teflide / Kirchner, Katharina / Yilmaz, Tuncay (2003): Die Motivation von DaF-Lernenden an Sprachlehrinstitutionen im Bielefelder Raum: Projektbeschreibung und erste Ergebnisse. In: Zeitschrift für Interkulturellen Fremdsprachenunterricht, 7/2/2003. Verfügbar unter: http://zif.spz.tudarmstadt.de/jg-07-2/beitrag/schlak1.htm_(16. 2. 2016).

Siebert, Horst (2006): Lernmotivation und Bildungsbeteiligung: Studientexte für Erwachsenbildung. Bielefeld. W. Bertelsmann Verlag.

Williams, Marion / Robert L. Burden / Safyia Al-Baharna (2001): Making sense of success and failure: The role of the individual in motivation theory. In: Dörney, Zoltán / Richard W. Schmidt (Hg.): Motivation and Second Language Acquisition. University of Hawaii. S. 171-184.

Eliška Dunowski

Goethe Institut Bremen

Bibliothekstr. 3

28359 Bremen

Eliska.Dunowski@goethe.de 